\title{
Serum squamous cell carcinoma antigen as an early indicator of response during therapy of cervical cancer
}

Stephanie Markovina ${ }^{\star}, 1,2$, Songyan Wang ${ }^{1}$, Lauren E Henke ${ }^{1}$, Cliff J Luke ${ }^{3}$, Stephen C Pak ${ }^{3}$, Todd DeWees ${ }^{1,2}$, John D Pfeifer ${ }^{2,4,5}$, Julie K Schwarz ${ }^{1,2}$, Weijun Liu1 ${ }^{1}$, Shuai Chen ${ }^{1}$, David Mutch ${ }^{2,5}$, Xiaowei Wang ${ }^{1,2}$, Matthew A Powell ${ }^{2,5}$, Barry A Siege ${ }^{2,6}$, Farrokh Dehdashti ${ }^{2,6}$, Gary A Silverman ${ }^{3}$ and Perry W Grigsby ${ }^{1,2,5,6}$ ${ }^{1}$ Department of Radiation Oncology, Washington University School of Medicine, St Louis, MO 63110, USA; ${ }^{2}$ Alvin J Siteman Cancer Center, Washington University School of Medicine, St Louis, MO 63110, USA; ${ }^{3}$ Department of Pediatrics, Washington University School of Medicine, St Louis, MO 63110, USA; ${ }^{4}$ Department of Pathology and Immunology, Washington University School of Medicine, St Louis, MO 63110, USA; ${ }^{5}$ Division of gynaecologic Oncology, Department of Obstetrics and Gynecology, Washington University School of Medicine, St Louis, MO 63110, USA and ${ }^{6}$ Division of Nuclear Medicine, Edward Mallinckrodt Institute of Radiology, Washington University School of Medicine, St Louis, MO 63110, USA

Background: Pretreatment serum squamous cell carcinoma antigen (SCCA) is a prognostic biomarker in women with cervical cancer. SCCA has not been evaluated as an early indicator of response to chemoradiation therapy (CRT). The molecular role of the two SCCA isoforms, SCCA1 (SERPINB3) and SCCA2 (SERPINB4), in cervical cancer is unknown. We hypothesised that changes in serum SCCA during definitive CRT predicts treatment response, and that SCCA1 mediates radiation resistance.

Methods: Patients treated with definitive CRT for cervical squamous carcinoma with serum SCCA measured were included. SCCA immunohistochemistry was performed on tumour biopsies. Post-treatment FDG-PET/CT, recurrence, and overall survival were recorded. Radiation response of cervical tumour cell lines after SCCA1 expression or CRISPR/Cas9 knockout was evaluated by clonogenic survival assay.

Results: Persistently elevated serum SCCA during definitive CRT was an independent predictor of positive post-therapy FDGPET/CT $(P=0.043)$, recurrence $(P=0.0046)$ and death $(P=0.015)$. An SCCA1-expressing vector increased radioresistance, while SCCA knock out increased radiosensitivity of cervical tumour cell lines in vitro.

Conclusions: Early response assessment with serum SCCA is a powerful prognostic tool. These findings suggest that escalation of therapy in patients with elevated or sustained serum SCCA and molecular targeting of SCCA1 should be considered.

The standard treatment for locally advanced cervical cancer is chemoradiation therapy (CRT). Patients with advanced disease and lymph node metastases are at risk for recurrence and dying from cervical cancer (Varia et al, 1998; Rose, 2003; Eifel et al, 2004; Kidd et al, 2010b). Prognostic biomarkers derived from positron emission tomography/computed tomography (PET/CT) with $2-\left[{ }^{18} \mathrm{~F}\right]$ fluoro-2-deoxy-d-glucose (FDG) include high tumour FDG uptake, large metabolic tumour volumes, and lymph node involvement at diagnosis (Grigsby et al, 2001; Xue et al, 2006; Kidd et al, 2007, 2010a). FDG-PET/CT metabolic response assessed three months after treatment is associated with outcomes (Schwarz et al, 2007), but a proportion of patients with complete metabolic

*Correspondence: Dr S Markovina; E-mail: smarkovina@wustl.edu

Received 18 July 2017; revised 25 September 2017; accepted 5 October 2017; published online 7 November 2017

(C) 2018 Cancer Research UK. All rights reserved 0007 - 0920/18 
response will still experience recurrence. Moreover, this assessment does not provide the opportunity to modify upfront therapy. FDG$\mathrm{PET} / \mathrm{CT}$ also remains a limited resource in locations where cervical cancer is most prevalent (Horton and Gauvreau, 2015). Prognostic tools realisable in limited-resource geographic areas could improve global outcomes for cervical cancer.

We have demonstrated that high pretreatment total serum squamous cell carcinoma antigen (hereafter referred to as just SCCA) is associated with recurrence, corroborating prior reports (Kato and Torigoe, 1977; Crombach et al, 1989; Hsieh et al, 1989; Bolli et al, 1994; Lin et al, 2000; Micke et al, 2005; Olsen et al, 2011). Similarly, failure of serum SCCA to normalise at the completion of CRT is a poor prognosis indicator (Ohara et al, 2002; Micke et al, 2005; Olsen et al, 2011). Chen et al (2017) demonstrated that tumour levels of SCCA protein correlate with response to neoadjuvant chemotherapy in patients undergoing surgery for cervical cancer. It is not currently known whether changes in serum SCCA during CRT indicate response to radiation. The relationship between serum and protein levels of SCCA within primary tumours has not been thoroughly reported. Moreover, evidence exists that SCCA in the serum contains two isoforms, SCCA1 (SERPINB3) and SCCA2 (SERPINB4). Both are members of the serine protease inhibitor (serpin) superfamily, with SCCA1 possibly playing a molecular role in resistance to common anti-cancer therapies (Suminami et al, 2000; Murakami et al, 2001; Hsu et al, 2009). A homologue of SCCA1 functions as a prosurvival factor in C. elegans by neutralising endogenous lysosomal proteases released upon cell stress (Luke et al, 2007). The role of SCCA1 in mediating tumour response to radiation is unknown.

We evaluated serum SCCA as a part of routine management of patients with cervical cancer treated with CRT at the time of diagnosis and weekly during treatment. Here we evaluate changes in serum SCCA levels as they relate to patient features, clinical outcomes including recurrence and overall survival, and FDG-PET results. In addition, we compared intracellular SCCA protein in pretreatment tumour specimens to circulating SCCA levels from corresponding serum samples. We used cervical tumour cell lines to directly test the role of SCCA1 in cervical tumour cell response to radiation.

\section{MATERIALS AND METHODS}

Patients and treatment. Prior approval from the Institutional Review Board with waiver of consent was obtained before reviewing medical records and existing specimens. Biopsy proven squamous cell carcinoma of the cervix and completed definitive CRT were inclusion criteria. The approach for definitive CRT at our institution has been previously described (Dyk et al, 2014). Briefly, patients were prescribed 50.4 Gray (Gy) external beam radiation therapy (EBRT) in $1.8 \mathrm{~Gy}$ fractions four days per week to the pelvis; para-aortic lymph node regions were included if involved on pre-treatment FDG-PET. Dose reduction of $10 \%$ in EBRT dose (prescribed dose $45 \mathrm{~Gy}$ ) was employed for elderly patients or those with very low body mass index $(<18.5$; Kizer et al, 2011). Intensity modulated radiation therapy (IMRT) and a split-pelvis or pseudo-step wedge technique were used with brachytherapy boost in six once-weekly fractions to a nominal high-dose rate (HDR) prescription of $6.5 \mathrm{~Gy}$ to point A (39 Gy total). Patients received concurrent weekly cisplatin at a dose of $40 \mathrm{mg} \mathrm{m}^{-2}$.

Serum SCCA. Serum SCCA assay was performed by ARUP National Reference Laboratory (Salt Lake City, UT, USA), using an enzyme-linked immunosorbent assay (ELISA; normal range 0.0$2.2 \mathrm{ng} \mathrm{ml}^{-1}$ ). Pretreatment serum SCCA was determined up to two months before the start of CRT and posttreatment within seven days of completing all radiation. On-treatment serum SCCA was binned into seven-day increments, and patients with at least three on-treatment serum SCCA levels (to allow a trend) were included for sub-analyses.

FDG-PET/CT. Pre- and posttreatment FDG-PET/CT was performed per routine clinical care. Standardised uptake value (SUV), a semiquantitative measure of radiotracer uptake, was used to calculate FDG uptake within the primary tumour (SUV= tissue radioactivity concentration $\left(\mathrm{nCi} \mathrm{ml}^{-1}\right) /[$ injected dose $(\mathrm{mCi}) /$ patient weight (g)) (Kidd et al, 2007). The primary tumour maximum SUV $\left(\mathrm{SUV}_{\max }\right)$ was determined as the maximum pixel SUV within a volume of interest (VOI) encompassing the tumour (Kidd et al, 2007). Metabolic tumour volume was calculated as the volume of pixels with tumour $\mathrm{VOI} \geqslant 40 \%$ of the $\mathrm{SUV}_{\max }$. For posttreatment FDG-PET/CT assessment, complete metabolic response was defined as resolution of abnormal FDG uptake at all sites and no new areas of abnormal FDG uptake, partial response as persistent abnormal uptake at initial sites of disease, and progressive disease as any new foci of abnormal uptake (Schwarz et al, 2007).

Outcome assessment. Recurrence was defined as new or progressive disease in any location (within the primary site, regional lymph nodes or distant metastases) as determined by clinical examination and/or imaging, with biopsy confirmation whenever possible ( $82 \%$ of recurrences were pathologically confirmed). Patient death was determined by review of the medical record, online obituary review and search of the Social Security Death Index. Positive post-therapy FDG-PET performed three months after completion of treatment was used as a separate outcome.

Immunohistochemistry. Formalin-fixed paraffin-embedded (FFPE) tumour specimens were marked for areas of tumour on haematoxylin and eosin stained slides. Triplicate $1.5 \mathrm{~mm}$ tumour cores were placed in a tissue microarray (TMA) using an MTA-1 Manual Tissue Arrayer (Beecher Instruments, Inc., Sun Prairie, WI, USA), and $6 \mu \mathrm{m}$ sections were deparaffinised, rehydrated, and after humidified heat antigen retrieval, stained with anti-SCCA1 and anti-SCCA2 antibodies ( $1: 25$, Santa Cruz Biotechnology Inc., Santa Cruz, California), and ImmunoCruz LSAB per the manufacturer's protocol (Santa Cruz Biotechnology, Inc.). Slides were digitised and analysed using the web-based Aperio eSlide Manager platform (Leica Biosystems, Buffalo Grove, IL, USA) in a blinded fashion by a pathologist with expertise in gynaecologic cancers (JP). Staining intensity and percent positive tumour cells were combined to an overall score as having absent, low or high staining. Subcellular localisation of staining was also noted.

Cell culture. All cell lines were obtained from ATCC and grown in monolayer at $37^{\circ} \mathrm{C} / 5 \% \mathrm{CO}_{2}$ in Dulbecco's modified Eagle's medium (DMEM) or Iscove's modified Dulbecco's medium (IMDM) supplemented with $10 \%$ foetal bovine serum, $0.1 \mathrm{mg} \mathrm{ml}^{-1}$ penicillin, 100 units per $\mathrm{ml}$ streptomycin, and $15 \mathrm{~mm}$ Hepes.

siRNA-mediated knockdown and generation of transgenic cell lines. HT3 cells were transfected with $30 \mathrm{~nm}$ siRNA (SCCA1 (h) sc- 40950 or scramble siRNA sc-37007, Santa Cruz Biotechnology, Dallas, TX, USA) with Lipofectamine RNAiMAX (Thermo Fisher Scientific, Waltham, MA, USA) per manufacturer's protocol. Knockdown was evaluated by qRT-PCR (primers in Supplementary Table 1) and WB after 24 and $48 \mathrm{~h}$ respectively. siRNA treated cells were seeded for radiation $24 \mathrm{~h}$ later. Clustered Regularly Interspaced Short Palindromic Repeats (CRISPR)/Cas9 mediated knockout of SERPINB3 was achieved using the LentiCRISPR V2 (Addgene 52961, Addgene, Cambridge, MA, USA) plasmid with either an empty guide RNA (gRNA) cassette or gRNA directed to the SERPINB3 sequence inserted as previously 
described (Shalem et al, 2014). Briefly, an all-in-one lentiviral plasmid, LentiCRISPR V2 (Addgene 52961), was engineered to house the SERPINB3-specific single guide RNA (sgRNA), $5^{\prime}$-CACCGGCCTGTACATCCTCCAGCG-3', identified using the publically available tool WU-CRISPR (available at http://crispr.wustl.edu/). $293 \mathrm{~T}$ cells were transduced with the plasmid for viral packaging, and HT3 and SW756 parent cell lines were infected with lentivirus. Puromycin-selected single cell clones were established, and knockout confirmed with WB analysis and genomic sequencing for SERPINB3 and potential off-target loci (Supplementary Table 1).

Western blot. Cells were lysed with Cell Lysis Buffer (Cell Signaling Technology, Danvers, MA) supplemented with proteinase/phosphatase inhibitors and PMSF. Equal parts protein $(25-30 \mu \mathrm{g})$ and Laemmli sample buffer (Santa Cruz Biotechnology, Dallas, TX, USA) were boiled at $95^{\circ} \mathrm{C}$ for 10 minutes and gel electrophoresed on 4-20\% gradient gels (Mini-Protean TGX, BioRad, Hercules, CA, USA), transferred to PVDF blot using the Trans-Blot Turbo Transfer system (Bio-Rad, Hercules, CA, USA), blocked with 5\% milk:TBS-Tween and incubated with 1:4000 antiSCCA1 antibody (NBP2, Novus International, Saint Louis, MO, USA) overnight at four degrees Celsius, 1:100000 anti-Actin (A5441, Santa Cruz Biotechnology, Dallas, TX, USA) or 1:2000 anti-GAPDH-HRP conjugated antibody (D16H11, Cell Signaling Technology, Danvers, MA, USA) for $2 \mathrm{~h}$ at room temperature. Anti-mouse or anti-rabbit HRP-conjugated secondary antibody was used for detection with ECL chemiluminescent reagent (GE Healthcare Life Sciences, Pittsburgh, PA, USA), visualised, and quantified using the Bio-Rad ChemiDoc MP imaging system and Image Lab software (Bio-Rad, Hercules, CA, USA).

Clonogenic survival assay. Five hundred cells per well were seeded in six-well plates $24 \mathrm{~h}$ prior to treatment with increasing doses of radiation $(2,4,6 \mathrm{~Gy} \times 1)$ at $300 \mathrm{cGy}$ per minute using the RS-2000 Biological System (Rad Source, Suwanee, GA, USA) and incubated for 1-3 weeks until control plates formed visible colonies $(\geqslant 50$ cells). Plates were fixed and stained with $0.5 \%$ crystal violet, $30 \%$ Methanol, $10 \%$ Acetic Acid, $60 \% \mathrm{ddH}_{2} \mathrm{O}$ for $30 \mathrm{~min}$, rinsed in tap water and air dried at room temperature. Surviving fraction was calculated as the number of colonies/(500 $\times$ plating efficiency).

Statistical analysis. Differences in survival and recurrence were tested using Kaplan-Meier analysis and log-rank testing. With various cut-points for serum SCCA published in the literature, we were motivated to identify the cut point for serum SCCA and time to normalisation that most effectively stratified our patient population for disease recurrence outcome. In order to achieve this, outcome oriented optimal cut-points for serum SCCA at diagnosis and time to normalisation of serum SCCA were identified based on the methodology of Contal and O'Quigley (Contal and O'Quigley, 1999). This methodology uses log rank test statistics with adjustments to preserve the type-I error rate. Odds ratio testing was used to determine an association between positive posttreatment FDG-PET and SCCA characteristics. Factors that were univariately significant for recurrence were considered for multivariate Cox-regression; however, given the known covariance of serum SCCA variables, separate models were built for each serum SCCA variable. Statistical significance threshold was $P<0.05$. All cell line experiments were performed in triplicate. Clonogenic cell survival curves were compared using linear repeated measures analysis.

\section{RESULTS}

Patients and outcomes. A total of 140 individual patients were treated with definitive CRT for cervical squamous carcinoma with
Table 1. Patient characteristics

\begin{tabular}{|l|c|}
\hline Age (mean, range) & 52 years, $25-83$ years \\
\hline Race & $93(66.4 \%)$ \\
\hline Caucasian & $38(27.2 \%)$ \\
Black & $6(4.3 \%)$ \\
Asian & $3(2.1 \%)$ \\
Hispanic & \\
\hline Stage & $13(9.4 \%)$ \\
\hline IB1 & $30(21.4 \%)$ \\
IB2 & $2(1.4 \%)$ \\
IIA & $57(40.7 \%)$ \\
IIB & $5(3.6 \%)$ \\
IIIA & $30(21.4 \%)$ \\
IIIB & $3(2.1 \%)$ \\
IVA & \\
\hline Node positive (on FDG-PET) & $82(58.6 \%)$ \\
\hline Pelvic LN & $24(17.1 \%)$ \\
PA LN & $140(100 \%)$ \\
Chemotherapy & \\
\hline Status at last follow-up & $86(61.4 \%)$ \\
\hline NED & $14(10 \%)$ \\
AWD & $36(25.7 \%)$ \\
DOD & $4(2.9 \%)$ \\
DICD & \\
\hline Abbreviations: AWD =alive with disease; DICD = died of intercurrent disease; DOD = died \\
of disease; LN =lymph node; NED =no evidence of disease; PA LN =para-aortic. \\
\hline \multicolumn{2}{|c|}{$\mid$} \\
\hline
\end{tabular}

FDG-PET/CT and had assessment of serum SCCA. Supplementary Figure 1 demonstrates the patients included for each analysis. All but three patients had serum SCCA measured before CRT. Of 100 patients with elevated serum SCCA $\left(>2.2 \mathrm{ng} \mathrm{ml}^{-1}\right)$, all had at least three (maximum six) on-treatment serum SCCA values and values at the completion of therapy available. FFPE specimens were available for 102 patients (73\%) in triplicate. Posttreatment FDGPET was obtained on 131 patients (94\%) included in the study, and obtained a median of 13 weeks after completion of therapy.

Cohort characteristics are displayed in Table 1. Pelvic lymph node involvement was detected by pre-treatment FDG-PET/CT in $58.6 \%$ of patients. All patients received concurrent cisplatin-based chemotherapy. Median time to completion of CRT was 48 days (range 43-95).

Median follow-up for all patients was 24.7 months, and 28.5 months for living patients. Crude recurrence rate was $36.4 \%$ with 19 pelvic only failures, 21 distant failures, and 11 pelvic and distant failures. Pathologic confirmation of recurrence with biopsy was obtained for 42 patients ( $82 \%$ of recurrences). At the time of last follow-up, 86 patients were alive and free of disease (61.4\%), 36 patients had died of cervical cancer $(25.7 \%)$, and four of intercurrent disease (2.9\%).

Serum SCCA. Pretreatment serum SCCA was obtained a median of 19 days prior to the start of EBRT (range; 3-37 days). Two patients had initial serum SCCA measured days two and three, respectively, of radiation therapy and were considered pretreatment values. The optimal cut-point for pretreatment serum SCCA identified as the most significant threshold for recurrence in our patient population (see Statistical Methods section) was found to be $16.1 \mathrm{ng} \mathrm{ml}^{-1}$. Patients with high pretreatment serum SCCA $\left(\geqslant 16.1 \mathrm{ng} \mathrm{ml}^{-1}\right)$ had significantly higher risk of positive posttreatment FDG-PET $(P=0.0063)$, disease recurrence $(P<0.0001)$, and death $(P<0.0001)$, compared to those with low pretreatment serum SCCA $\left(<16.1 \mathrm{ng} \mathrm{ml}^{-1}\right)$, Table 2 and Figure 1A and B. In patients with elevated pre-treatment SCCA $\left(\geqslant 2.2 \mathrm{ng} \mathrm{ml}^{-1}\right)$ failure of serum SCCA to normalise $\left(\leqslant 2.2 \mathrm{ng} \mathrm{ml}^{-1}\right)$ by the completion of therapy was associated with increased risk of recurrence 
$(P=0.016)$, death $(P=0.006)$, and positive post-therapy FDG-PET $(P=0.049)$, Figure $1 \mathrm{C}$ and $\mathrm{D}$, and Table 2.

Serum SCCA decreased over the course of CRT, with patients who experienced recurrence having significantly higher serum SCCA during the first 2 weeks of CRT (Figure 2A). In patients with elevated pre-treatment SCCA ( $\geqslant 2.2 \mathrm{ng} \mathrm{ml}^{-1}$ ) failure of SCCA to normalise by the 27th day of CRT was identified as the optimal time cut-point for prediction of recurrence and was an early indicator of increased risk of positive posttreatment FDG-PET $(P=0.043)$, recurrence $(P=0.0046)$, and death $(P=0.015)$, Table 2 and Figure $2 \mathrm{~B}$ and $\mathrm{C}$. Patients whose serum SCCA normalised by the 27th day of CRT and whose posttreatment FDG-PET was negative had an especially low risk of recurrence (Figure 2D).

A multivariate model was constructed from factors found to be associated with recurrence on univariate analysis, including FIGO stage, lymph node status (positive or negative by FDG-PET/CT), serum SCCA at diagnosis, serum SCCA normalisation by the completion of therapy, and time to normalisation of serum SCCA. Age, use of chemotherapy and other factors were not significant on univariate analysis and thus excluded from multivariate models.

Table 2. Odds ratio for positive post-therapy FDG-PET and serum SCCA metrics in patients with pre-treatment serum $S C C A \geqslant 2.2 \mathrm{n} \mathrm{ml}^{-1}$

\begin{tabular}{|l|c|c|c|}
\hline Serum SCCA metric & Odds ratio & $\mathbf{9 5 \% ~ C l}$ & $\boldsymbol{P}$ Value \\
\hline $\begin{array}{l}\text { Pretreatment } \\
\text { SCCA } \geqslant 16.1 \mathrm{ng} \mathrm{ml}^{-1}\end{array}$ & 4.2 & {$[1.5-11.76]$} & 0.0063 \\
\hline SCCA normalised by COT = No & 2.95 & {$[1.0003-8.7]$} & 0.049 \\
\hline $\begin{array}{l}\text { SCCA normalised by } 27 \\
\text { days = No }\end{array}$ & 3.1 & {$[1.039-9.25]$} & 0.043 \\
\hline Abbreviation: COT = completion of therapy. & \\
\hline
\end{tabular}

A

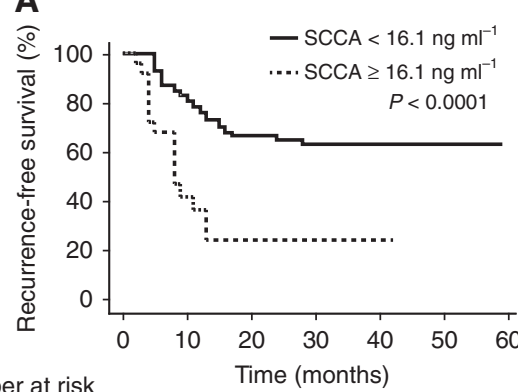

Number at risk

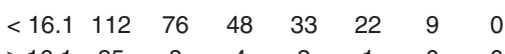

$\begin{array}{llllllll}\geq 16.1 & 25 & 8 & 4 & 2 & 1 & 0 & 0\end{array}$

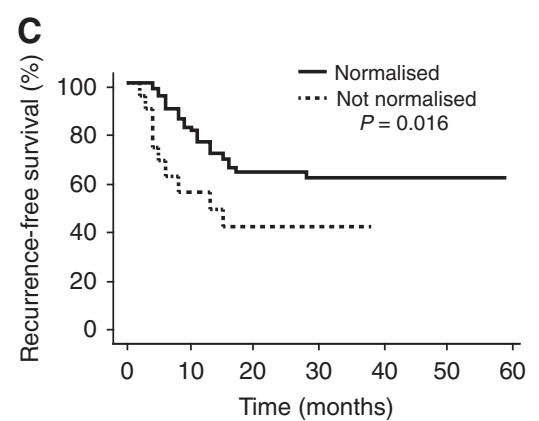

Number at risk $\begin{array}{lccccccc}\text { Normalised } & 81 & 54 & 33 & 26 & 18 & 7 & 0 \\ \text { Not normalised } & 19 & 8 & 5 & 2 & 0 & 0 & 0\end{array}$
Given inherent covariance of serum SCCA variables, individual models were built for each of these variables. Both serum SCCA at diagnosis $\geqslant 16.1 \mathrm{ng} / \mathrm{mL}$ and failure of SCCA to normalise by the completion of therapy remained significant on multivariate analysis as did positive lymph nodes (Supplementary Table 2).

SCCA immunohistochemistry. We next determined the relationship between serum levels of SCCA and intracellular tumour protein levels. IHC for SCCA1 and SCCA2 on the pretreatment tumour TMA was interpretable for 72 tumours. Staining for SCCA1 and SCCA2 was largely concordant between the samples. Staining ranged from absent to high (Figure 3A) and was correlated with pretreatment serum SCCA from the same patient $\left(P=0.004\right.$, Figure 3B). Tumour size, $\mathrm{SUV}_{\max }$, FIGO Stage, and lymph node status did not correlate with staining intensity (data not shown). The pattern of cellular staining (cytoplasmic, nuclear, perinuclear, or some combination) was variable (Supplementary Figure 2), and had no obvious relationship to serum SCCA.

Modulation of SCCA and effect on radiation sensitivity in vitro. Evaluation of a panel of cervical tumour cell lines showed variable levels of endogenous steady state SCCA1 protein by WB and variable radiation resistance by clonogenic cell survival assay (Figure 3C). We found that expression of GFP-taggedSCCA1 in SW756 cells, which are relatively radioresistant and have relatively low levels of endogenous SCCA resulted in increased clonogenic survival following radiation (Figure 3D). siRNAmediated knockdown of SCCA1 in HT3 cells which are radioresistant and have high SCCA1 protein resulted in decreased mRNA transcript, 2-fold lower protein levels (Supplementary Figure 2A), and increased radiation sensitivity compared to scramble-siRNA $(P=0.002$, Supplementary Figure $2 \mathrm{~B})$. Because maximum efficiency of knockdown resulted in only $50 \%$ protein

\section{B}

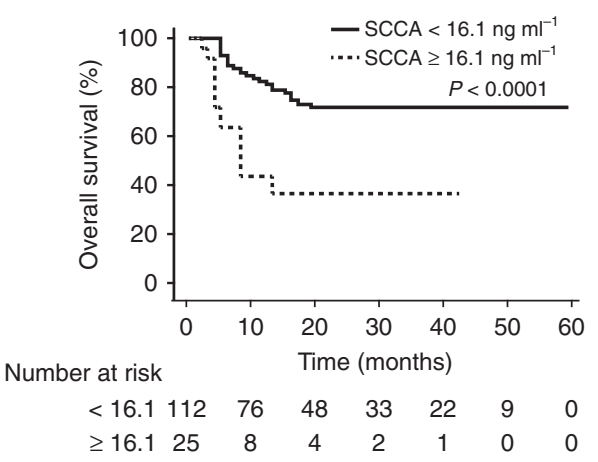

D

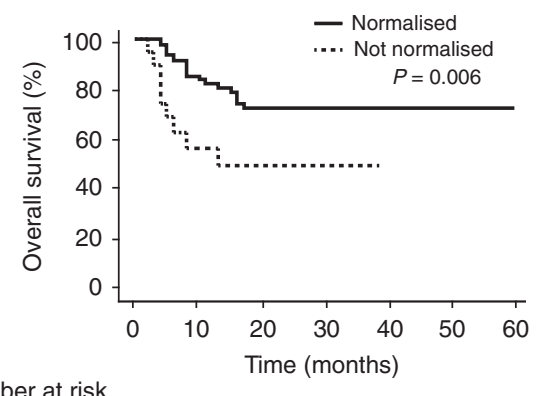

Number at risk

$\begin{array}{llllllll}\text { Normalised } & 81 & 54 & 33 & 26 & 18 & 7 & 0\end{array}$

Not normalised $\begin{array}{lllllll}19 & 8 & 5 & 2 & 0 & 0 & 0\end{array}$

Figure 1. Serum SCCA prognostic value. Kaplan-Meier plot of (A) recurrence-free survival and (B) overall survival in all patients $(n=137)$ with serum SCCA $\geqslant 16.1 \mathrm{ng} / \mathrm{ml}$ at diagnosis compared to those with serum SCCA $<16.1 \mathrm{ng} \mathrm{ml}^{-1}$. Kaplan-Meier plot of (C) recurrence-free survival and (D) overall survival in patients with pre-treatment serum SCCA $>2.2 \mathrm{ng} / \mathrm{ml}(n=100)$ that did or did not normalise $\left(\leqslant 2.2 \mathrm{ng} \mathrm{ml}{ }^{-1}\right)$ by the completion of therapy (COT). 
A

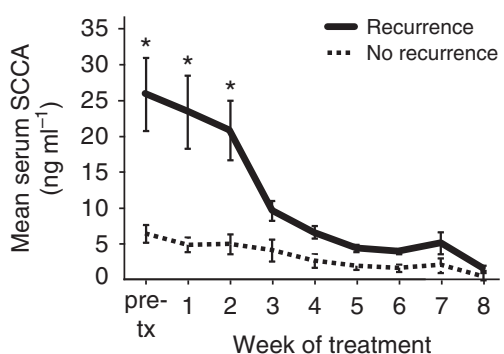

Number of values

$\begin{array}{llllllllll}\text { Recurrence } & 53 & 27 & 40 & 40 & 45 & 44 & 52 & 15 & 4\end{array}$ $\begin{array}{llllllllll}\text { No recurrence } & 80 & 44 & 68 & 68 & 65 & 63 & 66 & 31 & 2\end{array}$

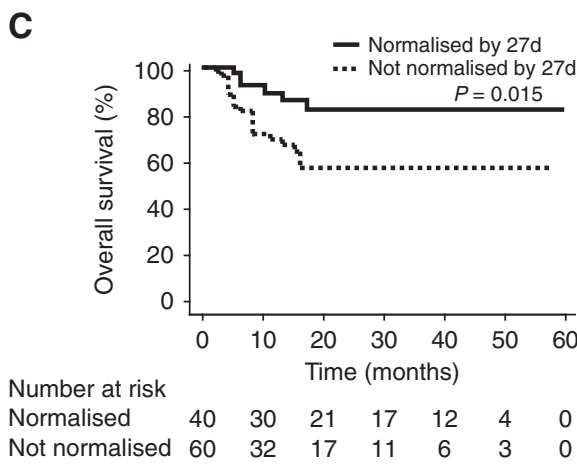

B

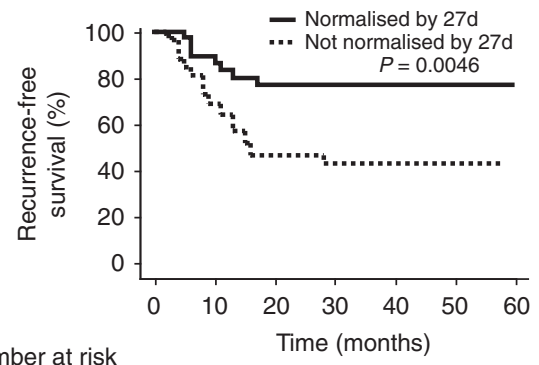

$\begin{array}{llllllll}\text { Normalised } & 40 & 30 & 21 & 17 & 12 & 4 & 0\end{array}$ $\begin{array}{llllllll}\text { Not normalised } & 60 & 32 & 17 & 11 & 6 & 3 & 0\end{array}$

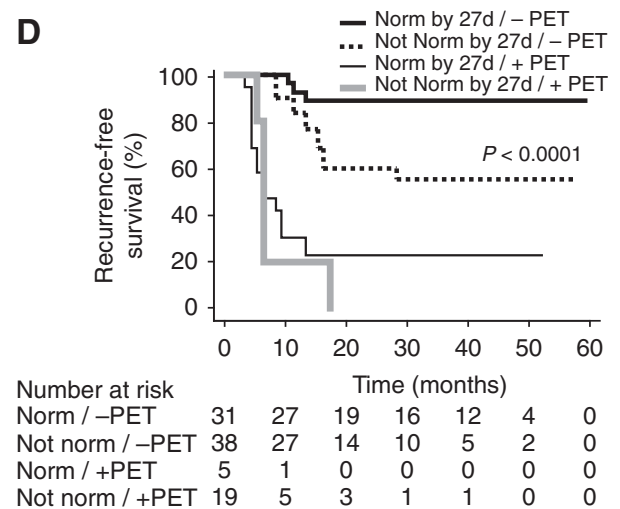

Figure 2. Changes in serum SCCA. (A) Mean serum SCCA values over the course of treatment split by recurrence. The number of individual values available for each time point are found below the graph. Asterisks indicate time points that were statistically different between recurrence and no recurrence groups. Kaplan-Meier plot of (B) recurrence-free survival and (C) overall survival of patients with pretreatment SCCA $>2.2$ ng $m$ l -1 $(n=100)$ and normalised serum SCCA by day 27 of CRT, versus those without. (D) Kaplan-Meier plot of recurrence free survival for patients with normalised serum SCCA by day 27, versus those without, split by positive or negative posttreatment FDG-PET (values available for 93 patients).

reduction, we used CRISPR-Cas9 with single guide RNA against SERPINB3, the gene encoding SCCA1, to generate SERPINB3 knock out tumour cell lines. This resulted in loss of SCCA1 protein by WB (Figure 3E), and demonstrated significantly increased radiation sensitivity of the SERPINB3 KO lines compared to parent or control cell lines (Figure 3F and G).

\section{DISCUSSION}

Women who experience recurrence of cervical cancer after definitive CRT have a grim prognosis and salvage therapy is largely ineffective (Eifel et al, 2006). Pre-treatment FDG-PET is used to guide nodal radiation treatment volumes; however, this method currently does not allow further individualisation of initial therapy based on risk. We sought a mechanism of identifying highrisk patients prior to the completion of CRT therapy, providing a possible opportunity to tailor initial treatment. SCCA is present in the serum in undetectable or low levels in the general population ${ }^{27}$ and elevated in many women with cervical cancer portending worse overall outcome (Crombach et al, 1989; Hsieh et al, 1989; Bolli et al, 1994; Lin et al, 2000; Ohara et al, 2002; Micke et al, 2005; Olsen et al, 2011). Here we confirm, in a population of women with squamous cervical cancer treated with uniform CRT, that pretreatment serum SCCA provides important prognostic information. We also demonstrate for the first time to our knowledge that this non-invasive serum test can serve as an early marker of response to CRT, of recurrence and of death, providing a window of opportunity to escalate therapy.

Elevated baseline serum SCCA levels can occur in a small percentage of women with cervical adenocarcinoma and adenosquamous carcinoma (our data not shown; Rose et al, 1993). To eliminate confounders secondary to histology, the current analysis was limited to squamous histology. Serum levels were obtained prospectively; however, not all patients had serum SCCA available for each week of radiotherapy limiting analysis of on-treatment SCCA. Similarly, not all patients had FFPE-tumour specimens available, limiting the current study.

While we showed that pretreatment serum SCCA values correlate with intra-tumoural SCCA by IHC, this measure represents only a single timepoint; therefore, we turned to cell line models to better understand the role of SCCA in tumour cell radiation resistance. Because the literature supports a possible role for SCCA1 in preventing stress-induced cell death and not SCCA2, we focused on SCCA1 for these initial mechanistic experiments. We observed that endogenous steady state SCCA1 was variable in a panel of cervical tumour cell lines, not unlike primary cervical tumours. We found that even a transient 50\% decrease in SCCA1 protein levels was sufficient to increase radiation sensitivity as evidenced by the gold standard clonogenic survival assay. SERPINB3 knockout significantly decreased clonogenic survival following radiation treatment of multiple cervical tumour cell lines. Moreover, transfection of an SCCA1-expressing vector into SW756 increased relative radiation resistance. The mechanisms are not certain, but several studies suggest that SCCA1 may protect tumours from the toxic effects of lysosomal cysteine proteases released upon exposure to noxious stimuli (Schick et al, 1998; Luke et al, 2007). Consistent with these findings, our data provide the first evidence to our knowledge that SCCA1 is directly involved in radiation resistance, and may explain its prognostic significance in cervical cancer.

'The Outback Trial,' an international randomised trial sponsored by the Australia New Zealand Gynaecological Oncology Group (ANZGOG), with participation of North American investigators from the NRG Oncology cooperative group, is currently testing the efficacy of adjuvant systemic chemotherapy 
A

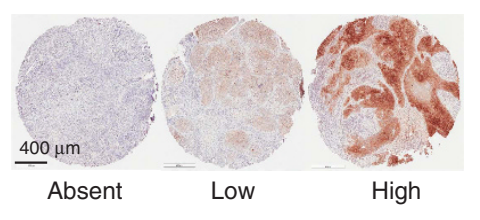

C
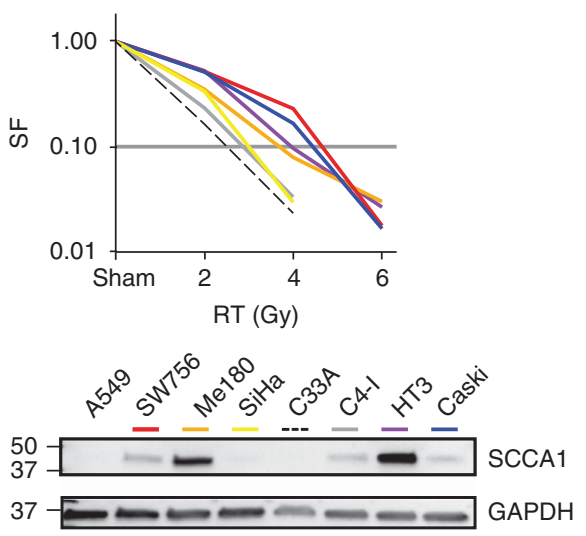

$\mathbf{E}$

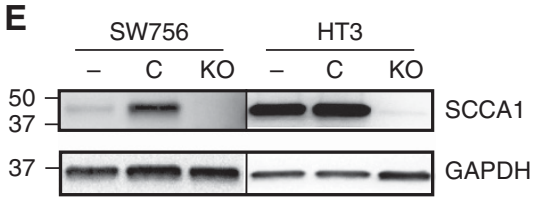

G

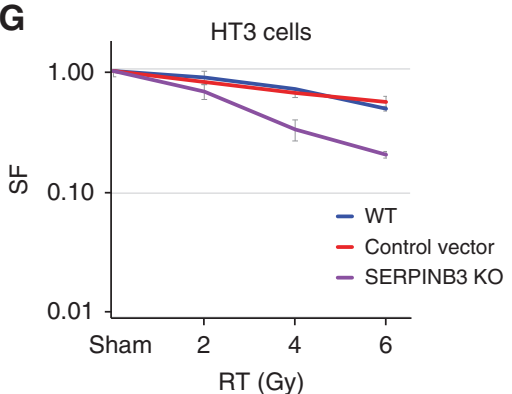

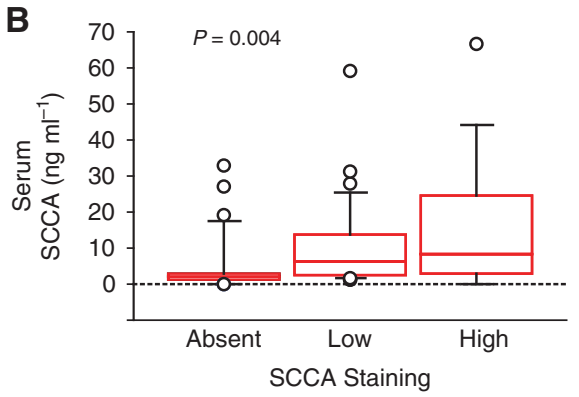

D
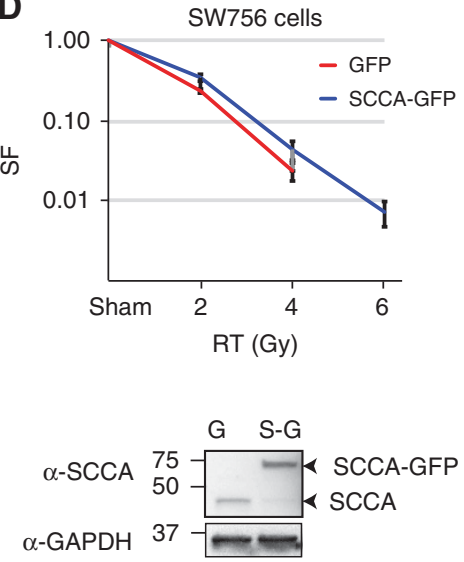

$\mathbf{F}$

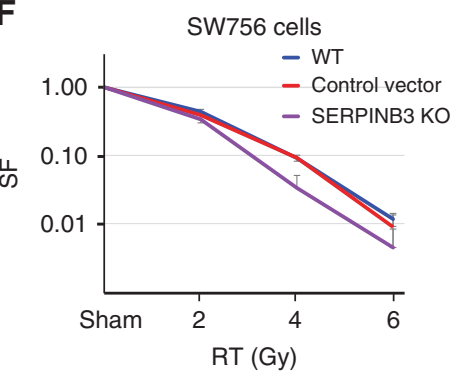

Figure 3. Intracellular SCCA1 protein. (A) Representative SCCA1 immunohistochemistry scoring, (B) box plot of SCCA1 staining score versus serum SCCA at diagnosis for the same patient, (C) ATCC panel of cervical tumour cell lines subject to Western blot analysis of SCCA1 and GAPDH and relative clonogenic survival of cervical tumour cell lines following increasing doses of RT delivered in a single fraction. (D) Western blot analysis of SCCA and GAPDH on whole cell lysates from SW756 cells engineered to express GFP only (G) or SCCA-GFP (S-G) construct and resultant relative clonogenic survival following increasing doses of single-fraction RT. (E) WB analysis of SCCA comparing CRISPR/Cas-9 control (C) gDNA or SERPINB3directed gDNA (KO) in SW756 and HT3 cells. ( $F$ and $\mathbf{G}$ ) Clonogenic survival of parent (WT), control and KO cell lines following increasing doses of RT.

after definitive CRT to reduce the risk of cervical cancer recurrence (Small). Eligibility criteria are broad and any benefit may be muted by the inclusion of women with a lower risk of recurrence. Employment of serum SCCA in this setting could provide a muchneeded tool to further stratify patients for likelihood of benefiting from such escalated initial therapy and should be considered in the design of future trials.

We also identified a group of patients with particularly low risk of recurrence-those with early serum SCCA normalisation and negative posttreatment FDG-PET-who are unlikely to benefit from additional therapy regardless of initial tumour stage.

Serum SCCA measured during post-treatment surveillance may allow early detection of recurrence with a lead-time of more than a year before development of symptoms or radiographic recurrence (Ohara et al, 2002). We are currently evaluating this possibility for more successful salvage of cervical cancer recurrence. SCCA, particularly SCCA1 may also be an important molecular factor involved in tumour cell resistance to radiation therapy. These data suggest that therapies targeting SCCA1 might increase radiation sensitivity and reduce the risk of recurrence. Our group is currently working to explore this possibility.

Elevated serum SCCA at diagnosis is a well-known but rarely utilised prognostic tool for women with cervical cancer. We provide the first evidence that failure of serum SCCA to fall into the normal range by the fourth week of definitive radiotherapy is associated with significantly increased risk of recurrence and death. 
This easily obtainable serum biomarker can be used to stratify patients for risk of recurrence and allow modification of initial treatment with the goal of improved outcomes.

\section{ACKNOWLEDGEMENTS}

The authors thank Jennifer German for administrative support and Anthony Apicelli for thoughtful discussion.

\section{CONFLICT OF INTEREST}

The authors declare no conflict of interest.

\section{REFERENCES}

Bolli JA, Doering DL, Bosscher JR, Day Jr TG, Rao CV, Owens K, Kelly B, Goldsmith J (1994) Squamous cell carcinoma antigen: clinical utility in squamous cell carcinoma of the uterine cervix. Gynecol Oncol 55: 169-173.

Chen P, Jiao L, Wang D-B (2017) Squamous cell carcinoma antigen expression in tumor cells is associated with the chemosensitivity and survival of patients with cervical cancer receiving docetaxel-carboplatinbased neoadjuvant chemotherapy. Oncol Lett 13: 1235-1241.

Contal C, O'Quigley J (1999) An application of changepoint methods in studying the effect of age on survival in breast cancer. Comput Stat Data Anal 30: 253-270.

Crombach G, Scharl A, Vierbuchen M, Würz H, Bolte A (1989) Detection of squamous cell carcinoma antigen in normal squamous epithelia and in squamous cell carcinomas of the uterine cervix. Cancer 63: 1337-1342.

Dyk P, Jiang N, Sun B, DeWees TA, Fowler KJ, Narra V, Garcia-Ramirez JL, Schwarz JK, Grigsby PW (2014) Cervical gross tumor volume dose predicts local control using magnetic resonance imaging/diffusionweighted imaging-guided high-dose-rate and positron emission tomography/computed tomography-guided intensity modulated radiation therapy. Int J Radiat Oncol Biol Phys 90: 794-801.

Eifel PJ, Jhingran A, Brown J, Levenback C, Thames H (2006) Time course and outcome of central recurrence after radiation therapy for carcinoma of the cervix. Int J Gynecol Cancer Off J Int Gynecol Cancer Soc 16: $1106-1111$.

Eifel PJ, Winter K, Morris M, Levenback C, Grigsby PW, Cooper J, Rotman M, Gershenson D, Mutch DG (2004) Pelvic irradiation with concurrent chemotherapy versus pelvic and para-aortic irradiation for high-risk cervical cancer: an update of radiation therapy oncology group trial (RTOG) 90-01. J Clin Oncol 22: 872-880.

Grigsby PW, Siegel BA, Dehdashti F (2001) Lymph node staging by positron emission tomography in patients with carcinoma of the cervix. J Clin Oncol 19: 3745-3749.

Horton S, Gauvreau CL (2015) Cancer in low- and middle-income countries: an economic overview. In Cancer: Disease Control Priorities. 3rd edn., Volume 3Gelband H, Jha P, Sankaranarayanan R, Horton S eds. The International Bank for Reconstruction and Development/The World Bank: Washington, DC, USA.

Hsieh CY, Chang DY, Huang SC, Yen ML, Juang GT, Ouyang PC (1989) Serum squamous cell carcinoma antigen in gynaecologic malignancies with special reference to cervical cancer. Taiwan Yi Xue Hui Za Zhi 88: 797-800.

Hsu K-F, Wu C-L, Huang S-C, Wu C-M, Hsiao J-R, Yo Y-T, Chen Y-H, Shiau A-L, Chou C-Y (2009) Cathepsin L mediates resveratrol-induced autophagy and apoptotic cell death in cervical cancer cells. Autophagy 5: 451-460.

Kato H, Torigoe T (1977) Radioimmunoassay for tumor antigen of human cervical squamous cell carcinoma. Cancer 40: 1621-1628.

Kidd EA, Siegel BA, Dehdashti F, Grigsby PW (2007) The standardized uptake value for F-18 fluorodeoxyglucose is a sensitive predictive biomarker for cervical cancer treatment response and survival. Cancer 110: 1738-1744.
Kidd EA, Siegel BA, Dehdashti F, Rader JS, Mutch DG, Powell MA, Grigsby PW (2010a) Lymph node staging by positron emission tomography in cervical cancer: relationship to prognosis. J Clin Oncol 28: 2108-2113.

Kidd EA, Siegel BA, Dehdashti F, Rader JS, Mutic S, Mutch DG, Powell MA, Grigsby PW (2010b) Clinical outcomes of definitive intensity-modulated radiation therapy with fluorodeoxyglucose-positron emission tomography simulation in patients with locally advanced cervical cancer. Int $J$ Radiat Oncol Biol Phys 77: 1085-1091.

Kizer NT, Thaker PH, Gao F, Zighelboim I, Powell MA, Rader JS, Mutch DG, Grigsby PW (2011) The effects of body mass index on complications and survival outcomes in patients with cervical carcinoma undergoing curative chemoradiation therapy. Cancer 117: 948-956.

Lin H, ChangChien CC, Huang EY, Tseng CW, Eng HL, Huang CC (2000) The role of pretreatment squamous cell carcinoma antigen in predicting nodal metastasis in early stage cervical cancer. Acta Obstet Gynecol Scand 79: $140-144$.

Luke CJ, Pak SC, Askew YS, Naviglia TL, Askew DJ, Nobar SM, Vetica AC, Long OS, Watkins SC, Stolz DB, Barstead RJ, Moulder GL, Brömme D, Silverman GA (2007) An intracellular serpin regulates necrosis by inhibiting the induction and sequelae of lysosomal injury. Cell 130: 11081119 .

Micke O, Bruns F, Schäfer U, Prott F-J, Willich N (2005) The impact of squamous cell carcinoma (SCC) antigen in patients with advanced cancer of uterine cervix treated with (chemo-)radiotherapy. Anticancer Res 25: 1663-1666.

Murakami A, Suminami Y, Hirakawa H, Nawata S, Numa F, Kato H (2001) Squamous cell carcinoma antigen suppresses radiation-induced cell death. Br J Cancer 84: 851-858.

Ohara K, Tanaka Y, Tsunoda H, Nishida M, Sugahara S, Itai Y (2002) Assessment of cervical cancer radioresponse by serum squamous cell carcinoma antigen and magnetic resonance imaging. Obstet Gynecol 100: 781-787.

Olsen JR, Dehdashti F, Siegel BA, Zighelboim I, Grigsby PW, Schwarz JK (2011) Prognostic utility of squamous cell carcinoma antigen in carcinoma of the cervix: association with pre- and posttreatment FDG-PET. Int $J$ Radiat Oncol Biol Phys 81: 772-777.

Rose PG (2003) Combined-modality therapy of locally advanced cervical cancer. J Clin Oncol 21: 211s-217s.

Rose PG, Baker S, Fournier L, Nelson BE, Hunter RE (1993) Serum squamous cell carcinoma antigen levels in invasive cervical cancer: prediction of response and recurrence. Am J Obstet Gynecol 168: 942-946.

Schick C, Brömme D, Bartuski AJ, Uemura Y, Schechter NM, Silverman GA (1998) The reactive site loop of the serpin SCCA1 is essential for cysteine proteinase inhibition. Proc Natl Acad Sci USA 95: 13465-13470.

Schwarz JK, Siegel BA, Dehdashti F, Grigsby PW (2007) Association of posttherapy positron emission tomography with tumor response and survival in cervical carcinoma. JAMA 298: 2289-2295.

Shalem O, Sanjana NE, Hartenian E, Shi X, Scott DA, Mikkelsen TS, Heckl D, Ebert BL, Root DE, Doench JG, Zhang F (2014) Genome-scale CRISPRCas9 knockout screening in human cells. Science 343: 84-87.

Small Jr W. RTOG | Clinical Trials | Study Number 1174. Available from http://rtog.org/ClinicalTrials/ProtocolTable/StudyDetails.aspx?study=1174 (accessed on 21 May 2015).

Suminami Y, Nagashima S, Vujanovic NL, Hirabayashi K, Kato H, Whiteside TL (2000) Inhibition of apoptosis in human tumour cells by the tumourassociated serpin, SCC antigen-1. Br J Cancer 82: 981-989.

Varia MA, Bundy BN, Deppe G, Mannel R, Averette HE, Rose PG, Connelly P (1998) Cervical carcinoma metastatic to para-aortic nodes: extended field radiation therapy with concomitant 5 -fluorouracil and cisplatin chemotherapy: a Gynaecologic Oncology Group study. Int J Radiat Oncol Biol Phys 42: 1015-1023.

Xue F, Lin LL, Dehdashti F, Miller TR, Siegel BA, Grigsby PW (2006) F-18 fluorodeoxyglucose uptake in primary cervical cancer as an indicator of prognosis after radiation therapy. Gynecol Oncol 101: 147-151.

This work is published under the standard license to publish agreement. After 12 months the work will become freely available and the license terms will switch to a Creative Commons AttributionNonCommercial-Share Alike 4.0 Unported License.

Supplementary Information accompanies this paper on British Journal of Cancer website (http://www.nature.com/bjc) 